\title{
In Vitro Analysis of Techniques that Alter the Surface Hardness of a Glass Ionomer Restorative Material
}

\author{
Riaan Mulder ${ }^{1}$, Naeemah Noordien ${ }^{2}$, Shaun Rossouw ${ }^{3}$, Luzaan van Zyl ${ }^{4}$
}

\begin{abstract}
Aim: The aim of this in vitro study was to compare the effect of different manipulation techniques on the surface hardness of ChemFil Rock glass ionomer.

Materials and methods: The changes in Vickers surface hardness (VH) of ChemFil Rock were evaluated after the application of five manipulation techniques and compared with one control group $(n=60)$. The manipulation techniques included: finger pressure set, electronic piezo producing a high frequency set, air piezo producing a low frequency set, heat-set achieved with a curing light and the last technique was a combination of electronic piezo followed by heat-set. Standard set was the control.

Results: The average surface hardness for the standard set was $49.5 \mathrm{VH}$. No statistical differences were demonstrated when the means were compared for finger pressure set $(49.2 \mathrm{VH})$ or the air piezo set $(48.49 \mathrm{VH})$ with standard set. The electronic piezo $(54.21 \mathrm{VH})$ and the heat-set $(57.5 \mathrm{VH})$ had an increased mean surface hardness when compared to other techniques. Heat-set had the highest surface hardness demonstrating a significant statistical difference when compared with standard set, finger pressure set, air piezo set as well as the combination of electronic piezo $(p<0.05)$.

Conclusion: The surface hardness of the glass ionomer cement $(\mathrm{GIC})$ material assessed in this study can be predictably improved by applying the heat-set technique. A command set can be achieved with the electronic piezo or the air piezo, however, the surface hardness will only increase with the use of the electronic piezo.

Clinical significance: The clinical advantage of using the air piezo as well as the electronic piezo technique would lie in preventing moisture contamination and dissolution of the GIC due to the command set effect of the ultrasonic vibrations within 15 seconds. The heat technique with the LED curing light will reduce the setting time to 90 seconds.
\end{abstract}

Keywords: Glass ionomer cement, Laboratory research, LED dental curing light, Surface microhardness, Ultrasonic scaler.

The Journal of Contemporary Dental Practice (2019): 10.5005/jp-journals-10024-2702

\section{INTRODUCTION}

Glass ionomer cement as restorative materials are advantageous in pediatric restorative dentistry. The main advantages of GIC include fluoride release up to $50 \mathrm{\mu g} / \mathrm{cm}^{2}$ and a chemical adhesion to the tooth structure. ${ }^{1-3}$ The linear coefficient of thermal expansion (CTE) of GIC is a clinically relevant property for temperature transfer to tooth structure, since it has been established as 10.2-11.4 CTE [ppm]. This is very similar to that of enamel (11.4 CTE [ppm]) and relatively close to dentine (8.3 CTE [ppm]), as measured at a temperature between $20^{\circ} \mathrm{C}$ and $60^{\circ} \mathrm{C} .{ }^{4,5}$ The two key disadvantages of GIC materials include low early strength and moisture sensitivity during the initial setting process. ${ }^{6,7}$

The setting reaction of GIC materials start to occur upon mixing the two components of the powder as the base and the liquid as the acidic component. The acid-base reaction continues until complete neutralization of the acidic liquid by the basic ions released from the powder is completed. ${ }^{8}$ Increasing the maturation reaction rate of the GIC will have a clinical advantage to reduce premature moisture contamination. Numerous techniques with ultrasonic scalers $s^{3,9,10}$ and curing units ${ }^{11-13}$ have been evaluated in literature to improve the properties of GIC. The faster setting time is one of the advantages of such techniques and enhances the resistance to water degradation in the initial \pm 10 minutes.

The literature on ultrasonic excitation and heat application to GIC showed a significant increase in the mechanical properties compared with the control materials. ${ }^{10,14-18}$ Heat generated from a curing light and the ultrasonic vibrations from an electronic
${ }^{1}$ Department of Restorative Dentistry, University of the Western Cape, Cape Town, South Africa

${ }^{2-4}$ Department of Pediatric Dentistry, University of the Western Cape, Cape Town, South Africa

Corresponding Author: Riaan Mulder, Department of Restorative Dentistry, University of the Western Cape, Cape Town, South Africa, Phone: +27 219373107, e-mail: rmulder@uwc.ac.za

How to cite this article: Mulder R, Noordien N, Rossouw S, et al. In Vitro Analysis of Techniques that Alter the Surface Hardness of a Glass Ionomer Restorative Material. J Contemp Dent Pract 2019; 20(12): 1362-1366.

Source of support: Nil

Conflict of interest: None

piezo has been reported to enhance the surface hardness of GIC. ${ }^{13}$ All the techniques evaluated in literature achieved improved material properties due to the manipulation of the salt bridge formation rate. ${ }^{10,19-21}$ Early application of heat after the placement of the restoration has been shown to improve the mechanical properties. ${ }^{10,17}$ The application of the electronic piezo resulted in an improved marginal adaptation of the GIC to the cavity wall. In turn, studies have demonstrated lower microleakage for the electronic piezo set vs the control group. ${ }^{21}$

Due to the varied methodologies used in literature related to the in vitro manipulation of GIC, a comparison of available techniques under similar conditions with the same GIC was 
required. The purpose of this study was therefore, to compare the changes in the surface hardness of a capsulated GIC after using different, readily available techniques from literature as a basis for the in vitro investigation collated in one study, to establish the true effect by using the same GIC material.

\section{Materials and Methods}

The in vitro study was approved by the ethics committee of The University of the Western Cape (BM/15/7/37).

\section{Study Design}

Ten samples were constructed for each experimental group from a capsulated GIC namely ChemFil Rock (Dentsply Sirona, Konstanz, Germany). A Teflon mould with a cylindrical space of $4 \mathrm{~mm}$ in diameter and $4 \mathrm{~mm}$ in height was used. Each of the moulds was randomly assigned to an experimental group and marked accordingly on the underside. The GIC material capsules were kept at room temperature to simulate the clinical scenario of storing GIC in the dental practice. The moulds were stored in a temperaturecontrolled incubator $\left(37^{\circ} \mathrm{C} \pm 1\right)$ prior to testing as to simulate the temperature in the oral environment. ${ }^{14} \mathrm{~A}$ single experienced operator performed the application of the manipulation techniques at a constant room temperature $\left(23 \pm 1^{\circ} \mathrm{C}\right)$ with a relative humidity of $50 \pm 5 \%{ }^{2,18}$ ChemFil Rock was mixed for 15 seconds at 4,000-4,500 oscillations per minute in an amalgamator (in accordance with manufacturer's instructions). Hand instruments were used to remove the excess GIC material from the mould that was slightly overfilled. A thin layer of petroleum jelly was applied to the back of the electronic- and air piezo tips to prevent adherence to the GIC when in contact with the piezo tip. A LED curing light (Bonart ART-L3, Taipei City, Taiwan) served as the heat source and was applied at a distance of $1 \mathrm{~mm}$ from the surface of the GIC.

\section{Division of Experimental Groups Control Group (Sts)}

Setting time of 3 minutes in accordance with the manufacturer's instructions.

\section{Electronic Piezo High Frequency Set (Ep)}

An electric piezo (Bonart ART-PB3, Taipei City, Taiwan) was set at $29 \mathrm{kHz}$ for 15 seconds $^{22}$ without water coolant. $^{23}$

\section{Air Piezo Low Frequency Set (Ap)}

The air piezo (Kavo Sonic flex 2000N, tip no 7, Biberach an der Riss, Germany) was set at $6 \mathrm{kHz}$ for 15 seconds $^{22}$ without water coolant. ${ }^{23}$

\section{Heat-set $(\mathrm{Ht})$}

The heat was provided by a LED curing light (Bonart ART-L3, Taipei City, Taiwan) at $1,450 \pm 50 \mathrm{~mW} / \mathrm{cm}^{2}$ for 40 seconds. $^{21}$

\section{Electronic Piezo Combined with Heat-set (Ep and $\mathrm{Ht}$ )}

The electronic piezo $(29 \mathrm{kHz})$ was applied to the surface of the GIC for 15 seconds without water coolant. This was followed immediately by heat provided by the LED curing light at 1,450 \pm $50 \mathrm{~mW} / \mathrm{cm}^{2}$ for 40 seconds.

\section{Finger Pressure Set (Fpre)}

The mould was placed on a desktop scale (Ohaus Precision Standard, Model TS400D, Ohaus Corp, Florham Park, NJ, USA) and $3 \mathrm{~kg} \pm 100 \mathrm{~g}\left(0.238 \mathrm{kgf} / \mathrm{mm}^{2}=2.3411 \mathrm{~N} / \mathrm{mm}^{2}\right)$ of pressure was applied with a latex-free gloved thumb covered with a thin layer of petroleum jelly. The pressure was maintained on the sample for 2 minutes immediately after GIC placement in the mould.

After completion of each technique, a layer of petroleum jelly was placed on the surface of the specimens to protect them against dehydration and moisture contamination during the initial setting. ${ }^{24}$ Each specimen was left for 6 minutes after the initial technique application (as per manufacturer's instructions) and then completely submerged in distilled water at $37^{\circ} \mathrm{C} \pm 1$.

\section{Surface Microhardness Analysis}

Forty-eight hours after the specimens were submerged in distilled water, the surface of the sample was smoothened using 2,500 grit silicon carbide paper, followed by 4,000 grit (3M, Massachusetts, USA). The polishing ensured an adjustment to the surface of \pm 100 $\mu \mathrm{m}$, as measured with a micrometer prior to the surface hardness evaluation. This simulates the restoration polishing that would occur in the clinical setting. ${ }^{14} \mathrm{~A}$ second and blinded operator completed the surface hardness evaluation with a Vickers hardness $(\mathrm{VH})$ indenter. The $\mathrm{VH}$ of the surface was determined with a Vickers diamond indenter (Zwick-Roell durometer, ZHV1/2 MicroVickers, Italy) set at HV0.5 (load of $500 \mathrm{gf}$ ) and a dwell time of 10 seconds. Three $\mathrm{VH}$ measurements were recorded $500 \mu \mathrm{m}$ apart on each sample to obtain a mean value per specimen. The mean values from the 10 samples per test group ${ }^{15,25,26}$ were calculated with the standard deviation (Fig. 1). The mean values of the $\mathrm{VH}$ measurements were converted to Logarithms (base e) and these values were noted as " $\log _{\mathrm{e}}$ (surface hardness)" for each test group. Analysis of variance (ANOVA) $t$ tests were carried out for statistical comparisons of the various values obtained from the $\log _{\mathrm{e}}$ (surface hardness), at a significance level of $p<0.05$ (Fig. 2).

\section{Results}

Figure 2 demonstrates the observed spread of the $\log _{e}$ (surface hardness) values. Ep as well as the Ep and $\mathrm{Ht}$ demonstrated the greatest spread. A Bartlett test of homogeneity of variances confirmed that a significant difference was present $(p<0.001)$ between the homogeneity of the various treatments.

Therefore, the Welch version of the ANOVA $t$ test allows for the appropriate evaluation of these differences in homogeneity between the manipulation techniques. When no overlap of the upper and lower limits occurs with the Welch version of the ANOVA

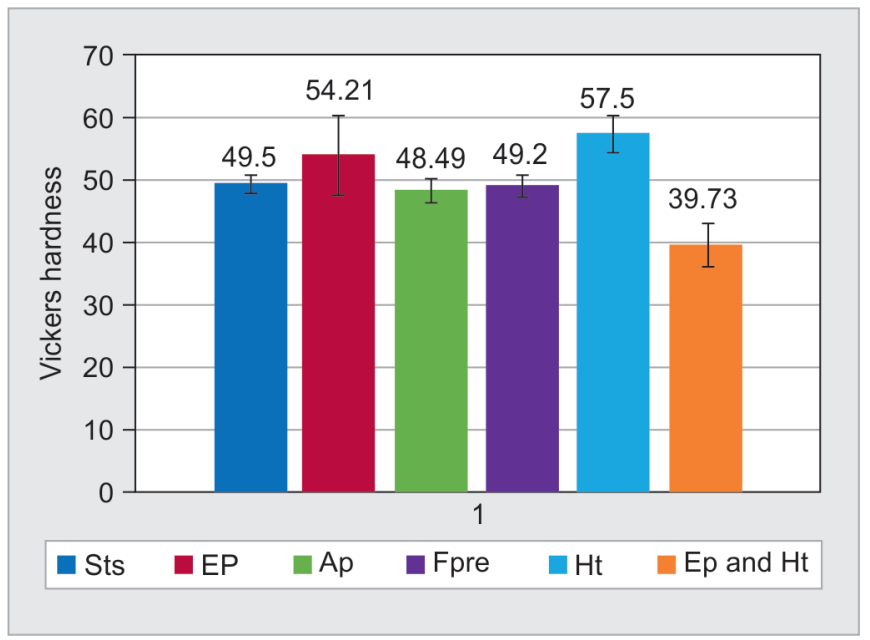

Fig. 1: Average of the $\mathrm{VH}$ with standard deviation for each technique 


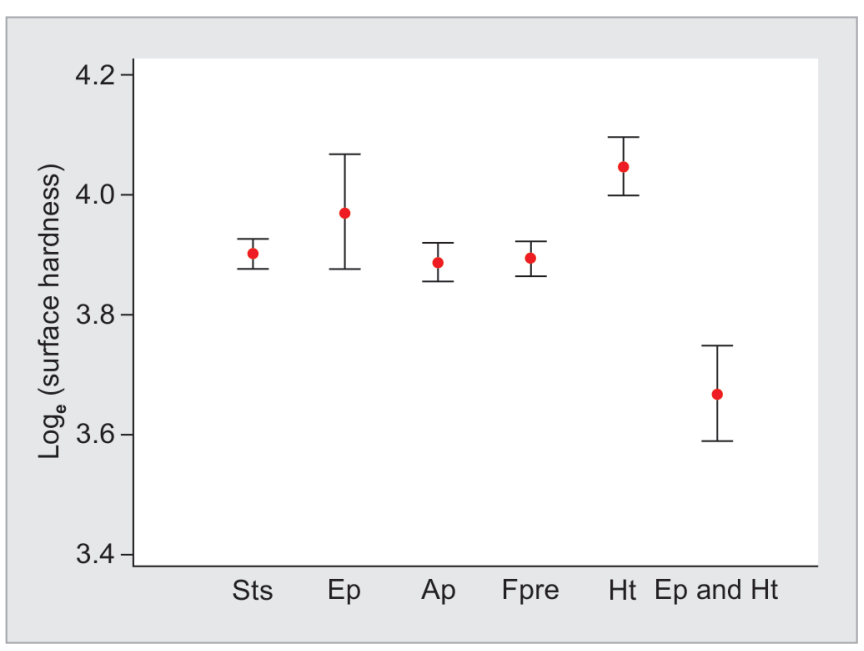

Fig. 2: $\log _{\mathrm{e}}$ (surface hardness) of the various techniques

$t$ test, it illustrates a significant difference between techniques at a level of $p<0.05$ (Fig. 2). There is therefore, no significant difference between the $\mathrm{VH}$ of the $\mathrm{Ht}$ and the Ep techniques in Figure 2. Only the $\mathrm{Ht}$ technique demonstrated an increased surface hardness that was significantly different when compared to the Sts. No significant difference was noted when applying the Fpre (49.2 VH) and the $\mathrm{Ap}(48.49 \mathrm{VH})$ techniques in comparison to Sts. Furthermore, the average of the $\mathrm{VH}$ of the $\mathrm{Ep}$ and $\mathrm{Ht}(39.73 \mathrm{VH})$ technique was statistically lower than Sts (49.5 VH). The technique that resulted in the greatest surface hardness was $\mathrm{Ht}$ technique (57.5 VH). The second-best technique was Ep (54.21 VH).

\section{Discussion}

The use of the various techniques was investigated with $\mathrm{VH}$ to establish the extent of the manipulation upon the "salt bridge" formation for the GIC material used in this study. The liquid phase of ChemFil Rock consists of polycarboxylic- and tartaric acid. The powder phase contains zinc modified fluoro-alumino-silicate glass filler particles. ${ }^{27}$ The resultant glass particle has been cited to be a calcium-aluminium-zinc-fluoro-phosphor-silicate glass. ${ }^{28}$ During the first mixing phase in the amalgamator, the polycarboxylic acid $\left(\mathrm{R}-\mathrm{COOH}^{-}\right)$in the liquid hydrate forms the glass particles of the powder. This is the start of the acid-base reaction and results in the exchange of protons from the glass filler particles causing the release of the cations $\left(\mathrm{Al}^{3+}, \mathrm{Ca}^{2+}, \mathrm{Sr}^{2+}, \mathrm{Zn}^{2+}\right)$. While this reaction takes place, the water content in the ChemFil Rock causes the polycarboxylic acid to neutralize and form a $\mathrm{R}-\mathrm{COO}^{-}$molecule. The cations $\left(\mathrm{Al}^{3+}, \mathrm{Ca}^{2+}, \mathrm{Sr}^{2+}, \mathrm{Zn}^{2+}\right)$ cross-link ionically to the $\mathrm{R}-\mathrm{COO}^{-}$ and then water is released during the binding of cations with the $\mathrm{R}-\mathrm{COO}^{-}$molecule. The application of ultrasonic excitation from $\mathrm{Ep}$ and the Ap techniques improves the reactivity of this afore mentioned process. ${ }^{19}$ This excitation causes the movement of the cations and the liquid of the ChemFil Rock to the surface, where the cations join with the neutralized $\mathrm{R}^{-\mathrm{COO}^{-}}$chains resulting in an accelerated $\mathrm{R}^{-\mathrm{COO}^{-}}$-cation salt bridge formation. This results in the rapid "command set" from Ep and the Ap techniques when compared to the $\mathrm{Ht}$, where no ultrasonic excitation was present. Command set refer to the ability of the clinician to induce a setting reaction, which occur earlier compared to when the material would have been left to set at its own speed. Barata ${ }^{20}$ cited that the use of ultrasonic excitation could provide the start of the command set.
The resultant surface hardness of the Ep in this in vitro study was greater, but not statistically different from the control (Sts) due to the surface hardness values having a greater standard deviation (Figs 1 and 2). Ultrasonic stimulation of both the Ep and Ap techniques therefore resulted in a rapid set (salt bridge formation) within 15 seconds of excitation.

The ultrasonic vibrations enhance the reaction rate of the salt bridge formation and improve the mechanical properties of GIC. ${ }^{10,19,22}$ Although the Ap ultrasonic vibrations at $6 \mathrm{kHz}$ resulted in a command set, it did not improve the surface hardness compared to the control (Sts). This result confirmed that when an ultrasonic device is used, the minimum setting of $17 \mathrm{kHz}^{22}$ or higher is required to obtain the additional increase in the surface hardness of GIC.

Gorseta ${ }^{21}$ concluded the addition of heat generated by the $\mathrm{Ht}$, Ap and the Ep techniques result in a relatively small temperature increase of $2-3^{\circ} \mathrm{C}$ on the surface of the restoration. In contrast, a temperature increase of $2.5^{\circ} \mathrm{C}$ was cited by Algera ${ }^{19}$ for $\mathrm{Ht}$ (produced by soldering iron with tip temperature of $70 \pm 2^{\circ} \mathrm{C}$ ) and $1^{\circ} \mathrm{C}$ for the Ep techniques during orthodontic bracket cementation. The temperature or ultrasonic vibrations transferred through the orthodontic bracket were considered sufficient to increase the reactivity of the GIC molecules to rearrange and achieve a greater zone of ionic exchange during salt bridge formation in the surface of the GIC, resulting in an increased bond strength of the cements. ${ }^{19}$ It is well recognized that high intensity curing lights are potentially dangerous, but a 1-2 mm layer of GIC has been shown to be sufficient for thermal isolation of the pulp. ${ }^{29}$ Therefore, the use of Ep followed by Ht should be safe for use with GICs and it does not increase the pulpal temperature above the critical temperature of $5.5^{\circ} \mathrm{C}$. It is thought that the $\mathrm{Ht}$ and the $\mathrm{Ep}$ result in decreased susceptibility of the restoration to hydrolysis in the early setting stage with the polycarboxylic acid are becoming more reactive. ${ }^{3,19}$ Furthermore, a more consistent and predictable surface hardness was achieved in the present in vitro study and a previously confirmed study by Menne-Happ and Ilie $^{14}$ where heat $(\mathrm{Ht})$ was generated by a LED curing light.

During the salt bridge formation there is loosely bound water present in the GIC. ${ }^{24}$ The decreased surface hardness observed with Ep combined with $\mathrm{Ht}(\mathrm{Ep}$ and $\mathrm{Ht}$ ) was visually explained by surface dehydration and crack formation of the loosely bound water. The small moisture droplets forming between the Teflon and the GIC material are seen under $10 \times$ stereo microscope magnification (Carl Zeiss AG, Stemi508 stereomicroscope, Oberkochen, Germany) (Fig. 3). Although the rise in temperature of the samples was not determined the movement of the moisture to the surface in the $\mathrm{Ep}$ and $\mathrm{Ht}$ technique was not present when Ep and the Ht were applied individually. This deduction of a temperature increase of combining Ep and $\mathrm{Ht}$ technique above that of the individual Ep and $\mathrm{Ht}$ alone was made, based on the combined $\mathrm{Ep}$ and $\mathrm{Ht}$ technique that resulted in small droplets of moisture becoming visible under magnification around the margin of the material.

The materials subjected to the finger pressure (Fpre) technique were standardized at $3 \mathrm{~kg} \pm 100 \mathrm{~g}\left(0.238 \mathrm{kgf} / \mathrm{mm}^{2}=2.3411 \mathrm{~N} / \mathrm{mm}^{2}\right)$ for 2 minutes to ensure a standardized pressure during the setting process. This standardization had to be ensured, since previous studies conducted on the application of intra-oral finger pressure during crown cementation resulted in the subjective application of forces. ${ }^{1,30}$ The surface hardness obtained from the Fpre group (49.2 $\mathrm{VH}$ ) was not significantly different from the control (49.5 VH) and the Fpre technique will not contribute to an increase in the surface hardness of GIC. 


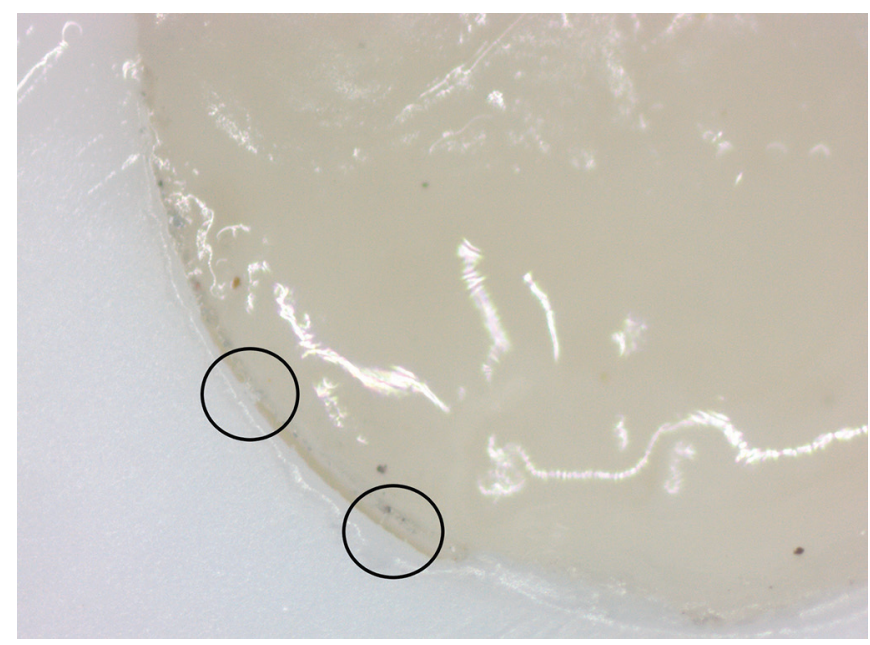

Fig. 3: Stereomicroscope image of water drops after Ep and $\mathrm{Ht}$ application

\section{Conclusion}

Because GIC materials are generally used in pediatric dentistry, the clinician could use the Ap and Ep techniques from this study to assist in the command set of the GIC material. The $\mathrm{Ht}$ technique with a LED dental curing light is the most effective technique to increase the surface hardness of the GIC. Clinicians should not combine the two techniques (Ep and $\mathrm{Ht}$ ) as this will lead to a significantly lower surface hardness.

\section{Clinical Significance}

The most challenging clinical scenarios where compliance and moisture control can be difficult, the clinician could make use of the piezo with the Ap and Ep techniques for the reduction in the setting time of the assessed GIC is desirable for pediatric restoration placement. The clinical advantage would lie in preventing moisture contamination of the GIC due to the command set effect of the ultrasonic vibrations. The use of the individual Ep and Ap techniques alone will induce the material to set within 15 seconds, preventing premature dissolution of the GIC and the Ep technique will improve the surface hardness to a greater extent than the Ap technique.

\section{LiMITATIONS}

Prewarming technique of glass ionomer material capsules were not assessed, since very few clinicians have access to a heating device like the Therma-Flo ${ }^{\mathrm{TM}}$ composite warmer (Vista dental, USA) or an appropriate water bath. The change in temperature at the bottom of the glass ionomer material during the use of each technique could be valuable for clinical recommendations to evaluate pulpal temperature changes for each technique. One capsulated glass ionomer material was assessed in this in vitro study.

\section{Acknowledgment}

This study formed part of a PhD study.

\section{References}

1. Black $S$, Amoore J. Measurement of forces applied during the clinical cementation of dental crowns. Physiol Meas 1993;14(3):387-392. DOI: 10.1088/0967-3334/14/3/018.
2. 9917-1:2007 I. ISO 9917-1:2007-Dentistry-Water-based cementsPart 1:Powder/liquid acid-base cements [Internet]. Iso.org. 2018 [cited 19 May 2018]. Available from: https://www.iso.org/standard/45818. html.

3. Khoroushi M, Karvandi TM, Sadeghi R. Effect of pre-warming and/ or delayed light activation on resin-modified glass ionomer bond strength to tooth structures. Oper Dent 2012;37(1):54-62. DOI: 10.2341/11-137-L.

4. Craig R. Restorative dental materials. 11th ed., London, UK: Mosby; 2002.

5. Esser M, Tinschert J, Marx R. Material characteristics of the hard tissues of bovine vs human teeth. Dtsch Zahnaerztl Z 1998;53(713):717.

6. Pelka M, Ebert J, Schneider $\mathrm{H}$, et al. Comparison of two- and three-body wear of glass-ionomers and composites. Eur J Oral Sci 1996;104(2):132-137. DOI: 10.1111/j.1600-0722.1996.tb00057.x.

7. Xie D, Brantley W, Culbertson B, et al. Mechanical properties and microstructures of glass-ionomer cements. Dent Mater 2000;16(2):129-138. DOI: 10.1016/S0109-5641(99)00093-7.

8. Sidhu S., Nicholson J. A review of glass-ionomer cements for clinical dentistry. J Funct Biomater 2016;7(3):16. DOI: 10.3390/jfb7030016.

9. Hübel $S$, Mejàre I. Conventional vs resin-modified glass-ionomer cement for Class II restorations in primary molars. A 3-year clinical study. Int J Paediatr Dent 2003;13(1):2-8. DOI: 10.1046/j.1365263X.2003.00416.x.

10. Kleverlaan C, van Duinen R, Feilzer A. Mechanical properties of glass ionomer cements affected by curing methods. Dent Mater 2004;20(1):45-50. DOI: 10.1016/S0109-5641(03)00067-8.

11. Hickel R, Manhart J, Garcia-Godoy F. Clinical results and new developments of direct posterior restorations. Am J Dent 2000;13: 41-54.

12. Hickel R, Kaaden C, Paschos E, et al. Longevity of occlusally-stressed restorations in posterior primary teeth. Am J Dent 2005;18(3):198-211.

13. Baloch F, Mirza A, Baloch D. An in vitro study to compare the microhardness of glass ionomer cement set conventionally vs set under ultrasonic waves. Int J Health Sci (Qassim) 2010;4(2):149-155.

14. Menne-Happ U, llie N. Effect of heat application on the mechanical behaviour of glass ionomer cements. Clin Oral Invest 2013;18(2): 643-650. DOI: 10.1007/s00784-013-1005-4.

15. O'Brien T, Shoja-Assadi F, Lea S, et al. Extrinsic energy sources affect hardness through depth during set of a glass-ionomer cement. J Dent 2010;38(6):490-495. DOI: 10.1016/j.jdent.2010.03.004.

16. Qvist V, Laurberg L, Poulsen A, et al. Eight year study on conventional glass ionomer and amalgam restorations in primary teeth. Acta Odont Scand 2004;62(1):37-45. DOI: 10.1080/00016350310008689.

17. Towler M. A preliminary comparison of the mechanical properties of chemically cured and ultrasonically cured glass ionomer cements, using nano-indentation techniques. Biomater 2001;22(11):1401-1406. DOI: 10.1016/S0142-9612(00)00297-0.

18. Twomey E, Towler M, Crowley C, et al. Investigation into the ultrasonic setting of glass ionomer cements part II setting times and compressive strengths. J Mater Sci 2004;39(14):4631-4632. DOI: 10.1 023/B:JMSC.0000034158.69184.84.

19. Algera $T$, Kleverlaan $C$, de Gee A, et al. The influence of accelerating the setting rate by ultrasound or heat on the bond strength of glass ionomers used as orthodontic bracket cements. Eur J Ortho 2005;27(5):472-476. DOI: 10.1093/ejo/cji041.

20. Barata $T$, Bresciani E, Adachi A, et al. Influence of ultrasonic setting on compressive and diametral tensile strengths of glass ionomer cements. Mater Res 2008;11(1):57-61. DOI: 10.1590/S151614392008000100011.

21. Gorseta K, Glavina D, Skrinjaric I. Influence of ultrasonic excitation and heat application on the microleakage of glass ionomer cements. Aust Dent J 2012;57(4):453-457. DOI: 10.1111/j.1834-7819.2012.01724.x.

22. Fagundesa T, Barataa J, Bresciania T, et al. Influence of ultrasonic setting on tensile bond strength of glass-ionomer cements to dentin. J Adhes Dent 2006;8(6):401-407.

23. Coldebella C, Santos-Pinto L, Zuanon A. Effect of ultrasonic excitation on the porosity of glass ionomer cement: a scanning electron 
microscope evaluation. Microsc Res 2010;74(1):54-57. DOI: 10.1002/ jemt.20873.

24. Hattab F, Amin W. Fluoride release from glass ionomer restorative materials and the effects of surface coating. Biomater 2001;22(12):1449-1458. DOI: 10.1016/S0142-9612(00)00253-2.

25. Ellakuria J, Triana R, Minguez N, et al. Effect of one-year water storage on the surface microhardness of resin-modified vs conventional glass-ionomer cements. Dent Mater 2003;19(4):286-290. DOI: 10.1016/ S0109-5641(02)00042-8.

26. Yap AU, Pek YS, Cheang P. Physico-mechanical properties of a fastset highly viscous GIC restorative. J Oral Rehab 2003;30(1):1-8. DOI: 10.1046/j.1365-2842.2003.01006.x.

27. Dentsply Sirona. Scientific Compendium of ChemFil Rock. [Internet]. 2011 [cited 19 May 2019]. Available from: https://www. dentsplysirona.com/content/dam/dentsply/pim/manufacturer/ Restorative/Direct_Restoration/Glass_lonomers/Classic_Glass_ Ionomers/ChemFil_Rock/ChemFil-Rock-nhg3c1d-scientificen-1402.

28. Zoergiebel J, llie N. An in vitro study on the maturation of conventional glass ionomer cements and their interface to dentin. Acta Biomater 2013;9(12):9529-9537. DOI: 10.1016/j.actbio.2013. 08.010 .

29. Kwon S, Park Y, Jun S, et al. Thermal irritation of teeth during dental treatment procedures. Restor Dent Endod 2013;38(3):105-112. DOI: 10.5395/rde.2013.38.3.105.

30. Zortuk M, Bolpaca P, Kilic K, et al. Effects of finger pressure applied by dentists during cementation of all-ceramic crowns. Eur J Dent 2010;4(4):383-388. DOI: 10.1055/s-0039-1697857. 